 \\ TATRA MOUNTaiNS \\ Mathematical Publications
}

\section{GAUSSIAN SEMIGROUPS ON HOMOGENEOUS BANACH SPACES}

\author{
MiloslaV DuchoŇ
}

\begin{abstract}
The object of this paper is a study of a Cauchy problem by Gaussian semigroups methods on the $2 \pi$-torus in the setting of homogeneous Banach spaces. The approach is similar to that in: [Butzer, P. L.-Berens, H. Semi-Groups of Operators and Approximation. Springer-Verlag, Berlin, 1967].
\end{abstract}

\section{Introduction}

Many problems, theorems and so on, in particular those where the methods of Fourier analysis may be applied to solve the Cauchy problem for the heat equation, are often stated in the setting that $X$ be one of the Banach spaces $C(0,2 \pi), C^{n}(0,2 \pi), L^{p}(0,2 \pi), 1 \leq p \leq \infty, M(0,2 \pi)$ and so on [1]. As for methods of Fourier analysis, the results obtained for the special spaces mentioned above may be very often extended for the so called homogeneous Banach spaces of functions and their dual spaces. Therefore many results in the theory of semigroups of operators obtained by means of Fourier analysis can be extended for the homogeneous Banach spaces, too.

The object of this paper is a study of a Cauchy problem by Gaussian semigroups methods on the $2 \pi$-torus in the setting of homogeneous Banach spaces. The approach is similar to that in 1 .

\section{Homogeneous Banach spaces}

Let $\mathbf{T}$ be the group of real numbers defined modulo $2 \pi, \mathbf{T}=R / 2 \pi Z$, $R$ - the reals, $Z$ - the integers. Equivalently, $\mathbf{T}$ is $[0,2 \pi]$ with 0 and $2 \pi$ identified or simply the torus. All functions and integrals are taken over the torus $\mathbf{T}$.

2000 Mathematics Subject Classification: 42A, 34B.

Keywords: homogeneous Banach space, Gaussian semigroup, Cauchy problem, Fourier analysis.

Supported by the Slovak grant agency VEGA, grant number 2/4137/07. 
The homogeneous Banach spaces over $\mathbf{T}$ are [2] 7] by definition, the linear subspaces $B(\mathbf{T})$ of $L^{1}(\mathbf{T})$ which are endowed with a norm \|\|$_{B}$ under which we have a Banach space such that

(i) $\|f\|_{1} \leq\|f\|_{B}$ for all $f \in B(\mathbf{T})$;

(ii) if $f \in B(\mathbf{T})$ and $x \in \mathbf{T}$, then $f_{x} \in B(\mathbf{T})$ and $\left\|f_{x}\right\|_{B}=\|f\|_{B}$, $\left[f_{x}(u)=f(u-x)\right]$;

(iii) $\lim _{u \rightarrow 0}\left\|f_{u}-f\right\|_{B}=0$ for all $f \in B(\mathbf{T})$.

Examples of homogeneous Banach spaces [4].

1. All spaces $L^{p}(\mathbf{T}), 1 \leq p<\infty$.

2. $C(\mathbf{T})$ - the space of all continuous $2 \pi$-periodic functions with the max norm.

3. $C^{n}(\mathbf{T})$ - the subspace of $C(\mathbf{T})$ of all $n$-times continuously differentiable functions with the common norm.

4. $L^{(1)}(\mathbf{T})$ - the Banach space of all functions $f$ on $\mathbf{T}$ which are absolutely continuous with the common norm.

5. $\operatorname{lip}_{q}(\mathbf{T}), 0<q \leq 1-$ ("small") Lipschitz spaces with the usual norm.

Note that $L^{\infty}(\mathbf{T})$-the space of all essentially bounded functions in $L^{1}(\mathbf{T})$ with the ess sup norm is not a homogeneous Banach space. The maximal homogeneous Banach space in it is the space $C(\mathbf{T})$.

Recall that a trigonometric polynomial on $\mathbf{T}$ is a function $a=a(t)$ defined on $\mathbf{T}$ by

$$
a(t)=\sum_{-n}^{n} a_{j} e^{i j t} .
$$

Denote by $p(\mathbf{T})$ the set of all trigonometric polynomials on $\mathbf{T}$.

Remark. If $B(\mathbf{T})$ is a homogeneous Banach space over $\mathbf{T}$, then for every $f \in B(\mathbf{T})$ and $n \in Z$ the function $x \rightarrow \hat{f}(n) e^{i n x}$ belongs to $B(\mathbf{T})$ [4, p. 6 , Lemma 1.9 and p. 17, Ex. 13]. It follows that if the Fourier-Lebesgue coefficient $\hat{f}(n)$ of $f$ is not zero, then the exponential $x \rightarrow e^{i n x}$ belongs to the space $B(\mathbf{T})$ for this $n \in Z$. So we may say that the exponential $x \rightarrow e^{i n x}$ belongs to $B(\mathbf{T})$ if there exists a function $f \in B(\mathbf{T})$ such that $\hat{f}(n)$ is not zero. With regard to this fact we will suppose, tacitly for the sake of simplicity, that $B(\mathbf{T})$ is a homogeneous Banach space containing all exponentials $x \rightarrow e^{i n x}, n \in Z$.

We may claim that trigonometric polynomials are dense in a homogeneous Banach space $B(\mathbf{T})$, namely, we shall need the following result [4, Th. 2.12].

Theorem. Let $B(\mathbf{T})$ be a homogeneous Banach space. For every $f \in B(\mathbf{T})$ we have $\sigma_{n}(f) \rightarrow f$ for $n \rightarrow \infty$, in the $B(\mathbf{T})$-norm. 
Recall that the Fejér sums of the Fourier series of a function $f$ are the trigonometric polynomials

$$
\sigma_{k}(f, t)=\sigma_{k}(t)=\sum_{-k}^{k}\left(1-\frac{|n|}{k+1}\right) \hat{f}(n) e^{i n t}, \quad k=1,2, \ldots
$$

\section{A Cauchy problem for homogeneous Banach spaces}

Let $B=B(\mathbf{T})$ be a homogeneous Banach space of functions $x \rightarrow f(x)$ over $\mathbf{T}$; for example one of the spaces $C(\mathbf{T}), L^{p}(\mathbf{T}), 1 \leq p<\infty$. We are interested in a solution of the following Cauchy problem:

Let $A$ be the operator defined by $A=\frac{d^{2}}{d x^{2}}$ on its domain

$$
D(A)=\left\{f \in B ; f, f^{\prime} \in A C(\mathbf{T}) \text { and } f^{\prime \prime} \in B\right\},
$$

where $A C(\mathbf{T})$ denotes the space of all absolutely continuous functions over $\mathbf{T}$.

Given an element $f \in B$, find a vector-valued function $s \rightarrow w(s)=w(s ; x)$ on $[0, \infty)$ to $B$ such that

(i) $s \rightarrow w(s)$ is strongly continuously differentiable in $(0, \infty)$;

(ii) for each $s>0, w(s) \in D(A)$ and $w^{\prime}(s)=A w(s)$;

(iii) $s-\lim _{s \downarrow 0} w(s, f)=f$.

The following characterization of domain of $A$ is an extension for homogeneous Banach spaces over $\mathbf{T}$ of a characterization given in [1] the proof being similar but for completeness we give it here.

Proposition 1. Let $B$ be a homogeneous Banach space over $\mathbf{T}$.

(a) $A$ is a closed linear operator with domain $D(A)$ dense in $B$.

(b) An equivalent characterization of the domain of $A$ is given by

$$
\left\{f \in B ; g \sim \sum_{k=-\infty}^{\infty} k^{2} \hat{f}(k) e^{i k x}, \quad g \in B\right\}
$$

and

$$
[A f]^{\wedge}(k)=-k^{2} \hat{f}(k), \quad k=0, \pm 1, \pm 2, \ldots, \quad f \in D(A) .
$$

$\left[g \sim \sum_{k=-\infty}^{\infty} k^{2} \hat{f}(k) e^{i k x}, g \in B\right.$ means that the series represents an element $g$ belonging to $B]$. 


\section{MILOSLAV DUCHOŇ}

Proof. (a) Obviously, $A$ is a linear operator. To prove that $A$ is closed, let $\left(f_{n}\right)_{n=1}^{\infty}$ be a sequence of elements in $D(A)$ such that $f_{n} \rightarrow f$ and $A f_{n}=f_{n}{ }^{\prime \prime} \rightarrow g$ in the $B$ norm. We have to prove that $f \in D(A)$ and $A f=f^{\prime \prime}=g$. The equality

$$
f_{n}(x+t)-f_{n}(x)-t f_{n}^{\prime}(x)=\int_{0}^{t}(t-u) f_{n}^{\prime \prime}(x+u) \mathrm{d} u, \quad n=1,2, \ldots
$$

holding for all $x$ and any real $t$ and properties of the homogeneous Banach space $B$ imply that $f_{n}{ }^{\prime}$ converges in norm for $n \rightarrow \infty$, say to the element $h$, and then $h$ and also $f$ belong to $A C(\mathbf{T})$ with $f^{\prime}=h$ and $h^{\prime}=g$. It follows that $A$ is closed. Clearly $p(\mathbf{T})$ is a subspace of $D(A)$ and since $p(\mathbf{T})$ is dense in $B$, we have immediately that $D(A)$ is a dense subset of $B$.

(b) If $f \in D(A)$ then by integration by parts we obtain

$$
[A f]^{\wedge}(k)=\frac{1}{2 \pi} \int_{0}^{2 \pi} e^{-i k x} f^{\prime \prime}(x) \mathrm{d} x=\frac{(i k)^{2}}{2 \pi} \int_{0}^{2 \pi} e^{-i k x} f(x) \mathrm{d} x=-k^{2} \hat{f}(k)
$$

for all $k=0, \pm 1, \pm 2, \ldots$, proving that $D(A)$ is a subset of the set of elements defined in (1). Conversely, if $f$ is a function in the set of (1) then we have for the Fejér means of the Fourier series

$$
f(x) \sim \sum_{k=-\infty}^{\infty} \hat{f}(k) e^{i k x}, \quad g(x) \sim \sum_{k=-\infty}^{\infty}\left(-k^{2}\right) \hat{f}(k) e^{i k x}
$$

that $A \sigma_{n}(f)=\left[\sigma_{n}(f)\right]^{\prime \prime}=\sigma_{n}(g)$. Since $A$ is closed and since $\sigma_{n}(f) \rightarrow f$ and $A \sigma_{n}(f)=\sigma_{n}(g) \rightarrow g$ as $n \rightarrow \infty$ in the norm of any homogeneous Banach space, it follows that $f \in D(A)$ and $A f=g$.

Proposition 2. Let $B$ be a homogeneous Banach space. The spectrum of $A$ is given by the point spectrum

$$
P_{\sigma}(A)=\left\{\lambda ; \lambda=-k^{2}, \quad k=0, \pm 1, \pm 2, \ldots\right\} .
$$

Also the resolvent set

$$
\rho(A)=\left\{\lambda ; \lambda \neq-k^{2}, \quad k=0, \pm 1, \pm 2, \ldots\right\}
$$

and the resolvent

$$
[R(\lambda ; A) f](x) \sim \sum_{k=-\infty}^{\infty} \frac{\hat{f}(k)}{\lambda+k^{2}} e^{i k x}, \quad f \in B .
$$

Moreover, for positive $\lambda, R(\lambda, A)$ has the representation

$$
[R(\lambda ; A) f](x)=\frac{1}{2 \pi} \int_{0}^{2 \pi} f(u) r(\lambda ; x-u) \mathrm{d} u
$$


with kernel

and

$$
r(\lambda ; u)=\frac{\pi}{\sqrt{\lambda}} \frac{\cosh \sqrt{\lambda}(u-\pi)}{\sinh \sqrt{\lambda} \pi}, \quad(0 \leq u<2 \pi)
$$

$$
\|R(\lambda ; A)\|=\frac{1}{\lambda}
$$

Proof. By part (b) of the foregoing proposition the homogeneous equation $\lambda f-A f=0$ is equivalent to

$$
\lambda \hat{f}(k)+k^{2} \hat{f}(k)=0, \quad k=0, \pm 1, \pm 2, \ldots
$$

Thus $\lambda$ belongs to the point spectrum $P_{\sigma}(A)$ if and only if $\lambda=-k^{2}$ and the eigenvectors corresponding to the eigenvalue $-k^{2}$ are given by $e^{i k x}$ and $e^{-i k x}$.

Consequently, for all $\lambda \neq-k^{2}, k$ integer, the operator $\lambda I-A$ has a unique inverse. Therefore, we obtain for an $f \in D(A)$ from $\lambda f-A f=g$, or equivalently from

$$
\lambda \hat{f}(k)+k^{2} \hat{f}(k)=\hat{g}(k), \quad k=0, \pm 1, \pm 2, \ldots
$$

that

$$
f(x)=\left[(\lambda I-A)^{-1} g\right](x) \sim \sum_{k=-\infty}^{\infty} \frac{\hat{g}(k)}{\lambda+k^{2}} e^{i k x} .
$$

Since the range $R(\lambda I-A)$ contains the trigonometric polynomials, it is a dense subset of $B$ and the representation of $(\lambda I-A)^{-1}$ given in the latter equation shows that its norm is bounded. Thus, according to the definition of the resolvent, $\lambda$ belongs to $\rho(A)$ and the resolvent $R(\lambda ; A)$ is given by (2).

It remains to prove the special representation of $R(\lambda ; A)$ for positive $\lambda$. Since the trigonometric series

$$
\sum_{k=-\infty}^{\infty} \frac{1}{\lambda+k^{2}} e^{i k x}
$$

is uniformly convergent for a fixed $\lambda$, it is the Fourier series of a function in $C(\mathbf{T})$. This function is given by $r(\lambda ; x)$ defined in (4), its Fourier coefficients $\hat{r}(\lambda ; k)$ being $1 /\left(\lambda+k^{2}\right), k=0, \pm 1, \pm 2, \ldots$ Thus

$$
\frac{1}{2 \pi} \int_{0}^{2 \pi} f(u) r(\lambda ; x-u) \mathrm{d} u=\sum_{k=-\infty}^{\infty} \frac{\hat{f}(k)}{\lambda+k^{2}} e^{i k x}
$$

which proves $(3)$. Since the kernel $r(\lambda ; \cdot)$ of the convolution integral is nonnegative, we have

$$
\|R(\lambda ; A) f\| \leq \hat{r}(\lambda ; 0)\|f\|=(1 / \lambda)\|f\| .
$$

The norm is in fact equal to $1 / \lambda$ as the particular function $f(x)=1$, for all $x$, shows. 


\section{MILOSLAV DUCHOŇ}

Now we can give the solution of the given Cauchy problem [1, Th. 1.5.3].

Theorem. Let B be a homogeneous Banach space. Then the given Cauchy problem has a unique solution

$$
w(t ; f)=W(t) f, \quad f \in B, \quad t \geq 0,
$$

where $W(t), 0 \leq t<\infty$, is strongly continuous contraction semigroup of operators in $L(B)$ generated by $A, A$ being its infinitesimal generator. The solution is the well-known periodic singular integral of Weierstrass

$$
[W(t) f](x)=\sum_{k=-\infty}^{\infty} e^{-k^{2} t} \hat{f}(k) e^{i k x}=\frac{1}{2 \pi} \int_{0}^{2 \pi} f(u) \vartheta_{3}(x-u ; t) \mathrm{d} u, \quad t>0,
$$

the kernel $\vartheta_{3}(\cdot ; t)$ being the so-called Jacobi theta function

$$
\sum_{k=-\infty}^{\infty} e^{-k^{2} t} e^{i k x}=\sqrt{\frac{\pi}{t}} \sum_{k=-\infty}^{\infty} \exp \left\{-\frac{(2 \pi k-x)^{2}}{4 t}\right\}, \quad t>0
$$

Further, $W(t), 0 \leq t<\infty$, forms a holomorphic semigroup.

Proof. By Proposition 2 all positive $\lambda$ belong to $\rho(A)$ and for positive $\lambda$, $\lambda R(\lambda ; A)$ is a contraction operator and thus $A$ generates a strongly continuous contraction semigroup of operators $W(t), 0 \leq t<\infty$, in $L(B)$. It then follows that the function $w(t ; f)=W(t) f$ on $[0, \infty)$ gives the solution of the given Cauchy problem for all $f \in D(A)$. In order to obtain an explicit representation for it compute the $k$ th Fourier coefficient of $W(t) f$ using a representation formula for $W(t)$ [1, Prop. 1.3.11]

$$
[W(t) f]^{\wedge}(k)=e^{-k^{2} t} \hat{f}(k), \quad t>0 ; \quad k=0, \pm 1, \pm 2, \ldots
$$

The Jacobi's theta function $\vartheta_{3}(x, t)$ defined in $(6)$ has the Fourier series

$$
\sum_{k=-\infty}^{\infty} e^{-k^{2} t} e^{i k x}, \quad t>0
$$

hence $W(t) f$ has the form as in (5). Further, (5) shows that $W(t)[B] \subset D(A)$ for all $t>0$ and since $f \star g \in B$ for all $f \in L^{1}$ and $g \in B$ we have for $f \in B$

$$
[A W(t) f](x)=\sum_{k=-\infty}^{\infty}\left(-k^{2}\right) e^{-k^{2} t} \hat{f}(k) e^{i k x}=\frac{1}{2 \pi} \int_{0}^{2 \pi} f(u) \vartheta_{3}^{\prime \prime}(x-u ; t) \mathrm{d} u, \quad t>0,
$$

and thus

$$
\|A W(t)\| \leq \int_{0}^{2 \pi}\left|\vartheta_{3}^{\prime \prime}(u ; t)\right| \mathrm{d} u \leq t^{-1}
$$




\section{GAUSSIAN SEMIGROUPS ON HOMOGENEOUS BANACH SPACES}

Hence the given semigroup is holomorphic and thus the function $t \rightarrow w(t)=$ $W(t) f$ gives a solution of the given problem for all $f \in B$.

It is easy to prove that the periodic singular integral of Weierstrass is the unique solution of the Cauchy problem for any homogeneous Banach space $B(\mathbf{T})$.

\section{REFERENCES}

[1] BUTZER, P. L.-BERENS, H.: Semi-Groups of Operators and Approximation. Springer-Verlag, Berlin, 1967.

[2] EDWARDS, R. E.: Fourier Series I (2nd ed.), Springer-Verlag, Berlin, 1979.

[3] EDWARDS, R. E.: Fourier Series II (2nd ed.), Springer-Verlag, Berlin, 1982.

[4] KATZNELSON, Y.: An Introduction to Harmonic Analysis (2nd ed.), Dower Publications, Inc., New York, 1976.

[5] SHILOV, G. E.: Homogeneous rings of functions, Usp. Mat. Nauk n. Ser. 653 (1951), no. 1, 91-137, (In Russian).

[6] SHILOV, G. E.: Homogeneous rings of functions, Amer. Math. Soc., Transl. 92 (1953), 392-453.

[7] SHILOV, G. E.: Mathematical Analysis, Fizmatgiz, Moscow 1961. (In Russian)

Received December 17, 2008
Mathematical Institute
Slovak Academy of Sciences
Štefánikova 49
SK-814 73 Bratislava
SLOVAKIA
E-mail: duchon@mat.savba.sk 